\title{
Acknowledgement to Reviewers of Cryptography in 2019
}

Cryptography Editorial Office

MDPI, St. Alban-Anlage 66, 4052 Basel, Switzerland

Published: 31 January 2020

The editorial team greatly appreciates the reviewers who have dedicated their considerable time and expertise to the journal's rigorous editorial process over the past 12 months, regardless of whether the papers are finally published or not. In 2019, a total of 26 papers were published in the journal, with a median time to first decision of 22 days and a median time from submission to publication of 56.5 days. The editors would like to express their sincere gratitude to the following reviewers for their generous contribution in 2019:

\begin{tabular}{ll} 
Aguilar, Leocundo & Gomaa, Ahmed \\
Alizadeh, Mojtaba & Gomez-Pau, Alvaro \\
Alvarez, Rafael & Graham, Scott \\
Avram, Camelia & Guenda, Kenza \\
Cambou, Bertrand & Halak, Basel \\
Cavenaghi, Marcos & Hashimoto, Yasufumi \\
Chan, Yu-Wei & Hong, Wien \\
Chandy, John A. & Iliyasu, Abdullah M. \\
Chen, Bin & Kang, James Jin \\
Chou, Jue-Sam & Kononovicius, Aleksejus \\
Ciatto, Giovanni & Kontogiannis, Sotirios \\
Cleaver, Gerald & Korodi, Adrian \\
Cope, Thomas & Kratzke, Nane \\
Craciunescu, Razvan & Krawec, Walter O. \\
Cruz, Homero Toral & Lara-Nino, Carlos Andres \\
De Castro, Alexandre & Leandro, Marín Muñoz \\
Delavar, Mahshid & Lee, Jeff \\
Duong, Dung Hoang & Li, Chengqing \\
Fan, Xin & Li, Yang \\
Farouk, Ahmed & Lim, Byung Hwa \\
Fine, Ben & Luo, Mingxing \\
Fujieda, Naoki & Mayberry, Travis \\
Ganji, Fatemeh (Saba) & Nogami, Yasuyuki \\
Gerault, David & Nuida, Koji \\
Gheorghiu, Alexandru & Ogiela, Marek R. \\
Gillis, Stacy & Pacyna, Piotr \\
Gołofit, Krzysztof & Parrilla, Luis \\
\hline & \\
\hline
\end{tabular}




\author{
Pathrose, Jerrin \\ Petzoldt, Albrecht \\ Piotrowski, Zbigniew \\ Pljonkin, Anton \\ Pricop, Emil \\ Pudukotai Dinakarrao, Sai Manoj \\ Righi, Rodrigo \\ Rønne, Peter Browne \\ Saito, Kumiko \\ Saracevic, Muzafer H. \\ Schmid, Giovanni \\ Senno, Gabriel Ignacio \\ Shaw, Harry \\ Shi, Hong-Ling \\ Singh, Mridula \\ Smith, Jad
}

Sodhro, Ali Hassan

Song, Yongsoo

Srikanth, R.

Stoyanov, Borislav

Suciu, George

Tlelo-Cuautle, Esteban

Udovenko, Aleksei

Uppu, Ravitej

Wang, Qiuhua

Weizman, Yoav

Wu, Hsin-Te

$\mathrm{Wu}$, Jun

Xie, Jiafeng

Xiong, Xiaoming

Zhang, Kejia

Zhu, Congxu

(C) 2020 by the author. Submitted for possible open access publication under the terms and conditions of the Creative Commons Attribution (CC BY) license (http://creativecommons.org/licenses/by/4.0/). 